\title{
L'endettement du Tiers Monde : analyses, objectifs et interventions de la Confédération suisse
}

Die Verschuldung der Dritten Welt: Analysen, Ziele und Interventionen der schweizerischen Eidgenossenschaft

\section{Antoine Brawand}

\section{CpenEdition}

\section{Journals}

Édition électronique

URL : http://journals.openedition.org/aspd/1342

DOI : 10.4000/aspd.1342

ISSN : 1663-9669

Éditeur

Institut de hautes études internationales et du développement

Édition imprimée

Date de publication : 1 janvier 1988

Pagination : 159-186

ISSN : 1660-5934

Référence électronique

Antoine Brawand, «L'endettement du Tiers Monde : analyses, objectifs et interventions de la Confédération suisse », Annuaire suisse de politique de développement [En ligne], 7| 1988, mis en ligne le 05 avril 2013, consulté le 08 septembre 2020. URL : http://journals.openedition.org/aspd/1342 ; DOI : https://doi.org/10.4000/aspd.1342 


\title{
L'endettement du Tiers Monde: analyses, objectifs et interventions de la Confédération suisse*
}

\author{
Antoine Brawand
}

Zusammenfassung. Dle Verschuldung der Dritten Welt: Analysen, Zlele und Interventionen der schweizerlschen Eidgenossenschaft

Welche Schlussfolgerungen haben die Bundesbehorden aus der Verschuldungskrise gezogen und in wieweit haben sie ihre Schlussfolgerungen in die Tat umgesetzt?

In einer 1984 veröffentlichten Analyse hatte der Bundesrat noch Tendenz, das Verschuldungsproblem als eine konjunkturelle Erscheinung anzusehen Heute erkennt er eindeutig deren strukturellen Charakter an. Der Bundesrat stellt fest, dass die von den Schuldnerländern durchgeführten Sanierung sprogramme nicht die enwarteten Ergebnisse erzielt haben und dass die Gläubigerlan der weder genügend

- Cette étude doit beaucoup à tous ceux qui ont bien voulu me faire part de leurs critiques et de leurs suggestions. Je leur en suis très reconnaissant. II va sans dire que j'assume l'entière responsabilité de la présentation des faits et de leur interprétation.

Une première version du texte, plus courte, a été présentée le 4 septembre 1987 au Groupe de travail «Affaires monétaires et financières internationales», à la Conférence générale de l'EADI (European Association of Development Research and Training Institutes), à Amsterdam.

Les documents utilisés pour cette étude sont antérieurs au 30 septembre 1987. 
Unterstüzung geboten, noch die unerlässlichen Anpassungen ihrer eigenen Wirtschaftsstrukturen vorgenommen haben. Er setzt sich weiterhin für die "BakerInitiative' ein.

Der Bund beteiligt sich an Schuldenkonsolidierungen für die ärmsten Länder, deren Bedingungen zwar jetzt etwas weniger hart sind, steht jedoch Vorschlägen einer Senkung der Zinssätze oder eines Schuldenerlasses ablehnend gegenaber. Hingegen hat er die Zahlungsbilanzhilfe verstärkt. Trotz Anerkennung der Mitverantwortung der Gläubigerländer haben die Bundesbehörden noch keine Politik zur Anpassung der Finanz- und Handelsflüsse der Schweiz festgelegt, welche die Wiederankurbelung der Wirtschaft der überschuldeten Länder erleichtern würde. Jedoch werden die Rufe nach einem Engagement der Schweiz, das dem Ausmass ihrer Verantwortungen entspricht, immer zahlreicher. Die Politik des Bundes wird sich daher sicherlich noch weiterentwickeln. 


\section{Introduction}

L'insolvabilité d'un nombre croissant de pays du Tiers Monde montre que la crise de l'endettement international ne pourra pas être résolue sans la mise en œuvre de politiques novatrices. Les autorités politiques des pays industrialisés, surtout dans ceux qui jouent un rôle commercial et financier en vue, devront prendre de nouvelles initiatives.

La Suisse détient une position privilégiée au sein de l'économie mondiale. Elle enregistre un excédent structurel de la balance des opérations courantes, particulièrement au niveau des opérations avec le Tiers Monde. Elle possède donc une bonne capacité de financement extérieur. Comment la Confédération suisse analyse-t-elle la crise de l'endettement, quelles solutions préconise-t-elle et quelles mesures prend-elle concrètement?

Jusqu'à présent, les autorités fédérales se sont relativement peu exprimées sur ces questions cruciales. Non-membre des institutions de Bretton Woods, mais entretenant d'excellentes relations avec elles, la Confédération suisse reste à l'arrière-plan dans les discussions internationales sur la crise de l'endettement. Cette discrétion, qui concorde avec les intérêts de la place financière suisse, est voulue. C'est ainsi qu'à la VIle session plénière de la CNUCED, à Genève, en juillet 1987, les délégués suisses ont fait des déclarations officielles sur les produits de base, sur le commerce international, et sur les pays les moins avancés, mais pas sur les ressources pour le développement et le problème de l'endettement. L'économie suisse pèse pourtant relativement plus dans ce dernier domaine: les créances suisses constituent environ 3,5\% de la dette extérieure du Tiers Monde, alors qu'au niveau des relations commerciales, les importations suisses représentent seulement $0,6 \%$ des exportations du Tiers Monde!

Cependant, la Confédération doit tenir compte de pressions et d'appels en faveur d'un engagement de la Suisse à la hauteur de ses moyens. Le Tiers Monde attend une participation effective au financement du développement et des mesures consistantes d'allègement de la dette extérieure. Les pays industrialisés et les organisations internationales demandent un partage équitable des charges. En Suisse même, le Conseil fédéral est de plus en plus souvent interpellé à propos de sa politique concernant l'endettement international.

Dans ce contexte, je n'aborderai pas seulement la politique actuelle de la Suisse; je tenterai aussi de repérer quelques évolutions possibles. II s'agit notamment d'examiner quelles leçons les autorités suisses ont tirées de la crise et comment elles pourraient les traduire en actes.

\section{Analyses et objectifs de la confédération suisse}

Les principales prises de position officielles figurent dans le Rapport sur les risques de l'endettement international (1) publié en 1984 en réponse à l'interpellation Reiniger (2), dans des exposés de hauts fonctionnaires de la Confédération (3) et du Président de la Banque nationale suisse (4), ainsi que dans une lettre du 
Conseiller fédéral Pierre Aubert à l'Action Place financière Suisse-Tiers Monde (5). On trouve aussi certaines prises de position dans les Messages du Conseil fédéral concernant la coopération au développement et la politique économique extérieure (6). Enfin, le Conseil fédéral a répondu le 16 septembre 1987 à l'interpellation Widmer (7). Des réponses doivent encore être données au postulat Longet (8) et à l' «Appel pour un commerce équitable avec le Tiers Monde» déposé par sept œuvres d'entraide (9).

\subsection{Quelle crise?}

Trois événements ont marqué l'analyse des autorités suisses: le moratoire mexicain en août 1982, qui a brusquement révélé les risques de l'endettement international; « ''initiative Baker », d'octobre 1985 et, enfin, l'aggravation récente de la crise en Amérique latine et en Afrique sub-saharienne.

\section{Les risques de l'endettement international}

Le Rapport sur les risques de l'endettement international publié en mars 1984 (1) réagit aux inquiétudes provoquées par la cessation de paiements mexicaine et par la série de moratoires qui lui a succédé ailleurs (2). Dans ce document, le Conseil fédéral distingue plusieurs causes de l'endettement du Tiers Monde:

- La récession économique qui a frappé l'économie mondiale, l'évolution défavorable pour les pays en développement des cours des matières premières, la montée du protectionnisme qui limite les débouchés commerciaux des exportateurs du Tiers Monde.

- Les politiques monétaires restrictives menées dans les pays industrialisés, qui ont provoqué la hausse des taux d'intérêt au début des années 1980.

- L'évolution des politiques suivies par les banques internationales: après avoir permis aux pays du Tiers Monde et d'Europe orientale de satisfaire sans peine et à des conditions avantageuses leurs besoins de financement externe, les banques ont restreint leurs prêts et raccourci les échéances.

- Certains pays débiteurs ont poursuivi trop longtemps leurs ambitieux programmes économiques sans prendre garde à la détérioration du contexte économique mondial. Ces pays ont parfois trop peu investi dans les branches exportatrices et dans la production de biens destinés à remplacer certaines importations. Des politiques souvent défavorables au secteur privé ont amplifié l'exode des capitaux.

Cette analyse officielle a le mérite de mettre largement en évidence la responsabilité commune des débiteurs et des créanciers. Aussi toute solution reposera-t-elle sur la coopération internationale: «Le processus s'étendra sur plusieurs années et son succès dépendra en fin de compte dans une large mesure 
de la capacité de l'économie mondiale à retrouver une croissance sans inflation et de la volonté des Etats industrialisés d'endiguer le protectionnisme et d'ouvrir davantage leurs marchés aux produits en provenance des pays débiteurs. (10)

Le rapport concluait sur un ton rassurant: « $A$ notre avis, il y a de bonnes chances de venir à bout de la crise internationale de l'endettement. Cette crise n'est pas due à un vice fondamental du système, mais à une surcharge temporaire.» (11) Le Conseil fédéral fondait son optimisme sur l'expansion qui s'était manifestée aux Etats-Unis en 1983 et qui paraissait alors susceptible d'entraîner une reprise généralisée.

\section{L'initiative Baker}

Les autorités suisses ont accueilli favorablement le discours du Secrétaire américain au Trésor, James Baker, prononcé en octobre 1985 devant l'Assemblée annuelle conjointe du FMI et de la Banque mondiale, à Séoul (12). Ce discours esquissait une stratégie coordonnée au niveau des institutions financières multilatérales, des banques privées et des Etats, fondée sur des politiques de croissance. Appelée bientôt «initiative» ou "plan Baker», cette stratégie qui devait contribuer à résoudre le problème de l'endettement comportait deux volets: I'octroi de nouveaux crédits à une quinzaine de pays en développement à revenu moyen lourdement endettés (apport d'environ 40 milliards de dollars en trois ans) et l'application par ceux-ci de programmes d'ajustement structurel comportant la libéralisation des structures économiques et favorisant le rapatriement volontaire de capitaux privés. De l'avis des autorités suisses, la mise en œuvre de cette stratégie aurait contribué à remédier à des difficultés déjà bien visibles à l'époque, notamment:

- Bien des pays débiteurs qui avaient redressé leur balance commerciale de façon spectaculaire à partir de 1982 y étaient parvenus en prenant des mesures d'austérité nuisibles à l'économie intérieure. La chute du niveau de vie et la détérioration de l 'appareil de production le montraient clairement.

- Simultanément, les flux financiers à destination des pays en développement continuaient à baisser dangereusement. Les taux d'intérêts réels restaient excessifs et l'exode des capitaux continuait. Les transferts financiers nets restaient négatifs.

- La croissance de l'économie mondiale ralentissait déjà, les prix des matières premières continuaient à baisser et le protectionnisme se renforçait.

Les autorités suisses voyaient aussi dans l'initiative Baker un signe que les Etats-Unis assumaient de nouveau leurs responsabilités de leader du système monétaire international. Le renforcement de la coopération internationale devait favoriser un transfert net de ressources vers les pays débiteurs, les réorienter vers une croissance soutenue dans le cadre de la division internationale du travail et faciliter le rétablissement de relations financières normales. 
On peut remarquer que cette analyse des autorités suisses semblait ignorer les critiques virulentes que l'initiative Baker a soulevées dans le Tiers Monde, notamment en Amérique latine. Certaines critiques se résument par la formule "Se développer pour pouvoir payern! (13)

\section{L'aggravation récente de la crise}

Le redressement prévu par les partisans des propositions Baker ne s'est pas produit. Au contraire, la baisse des cours des matières premières, la chute brutale du prix du pétrole, certaines mesures protectionnistes de la part des pays industrialisés et la diminution massive des crédits bancaires ont aggravé la situation de nombreux pays, particulièrement en Amérique latine et en Afrique subsaharienne (14).

Les banques, qui avaient assez bien accueilli le discours de J. Baker, n'ont pas donné l'appui financier attendu de leur part (20 milliards de dollars en trois ans). En 1985 , les prêts nets des banques aux quinze pays visés par l'initiative Baker avaient encore augmenté de 0,8 milliards de dollars; en 1986, ils ont par contre baissé de 2,8 milliards! Hormis les crédits à l'exportation garantis, les banques ont virtuellement arrêté l'octroi de crédits «volontaires». Les nouveaux prêts qu'elles ont consentis l'ont généralement été dans le cadre d'opérations concertées sur le plan international (15).

Simultanément, elles ont fortement accru leurs provisions pour créances douteuses. Les banques allemandes, françaises et suisses ont été parmi les premières à prendre de telles mesures. La réglementation bancaire les y incitait. Les banques américaines, britanniques et japonaises font désormais de même. En prenant ouvertement ces précautions, elles montrent que le risque de ne plus pouvoir récupérer l'intégralité des créances a augmenté. Par contre, l'accumulation des provisions renforce leur situation financière et leur capacité de négociation. Dans ces conditions, elles semblent pouvoir se passer désormais du plan Baker et des tentatives actuelles de relance de ce plan (16).

Dans sa réponse du 16 septembre 1987 à l'interpellation Widmer, le Conseil fédéral constate bien l'aggravation récente des problèmes de l'endettement. II affirme néanmoins: "Le système financier international semble toutefois être mieux à même qu'en 1982 de relever les défis auxquels nous sommes confrontés aujourd'hui». En effet, l'augmentation considérable des provisions pour créances douteuses «ont sans doute renforcé la sécurité du système financier international.» (17)

La réponse du Conseil fédéral contient aussi quelques informations sur la situation des banques suisses. Leurs avoirs dans les pays lourdement endettés "Ont régressé tant en chiffres absolus que par rapport au total de leur actif depuis le début de la crise de l'endettement... Alors qu'en 1982 déjà, l'ensemble des crédits à haut risque de notre système bancaire était inférieur à ses fonds propres - compte non tenu des réserves latentes disponibles et des provisions exigées par la Commission fédérale des banques... - cette proportion s'est encore fortement améliorée jusqu'à fin 1986. " (17) Après avoir montré les causes de cette «évolution 
favorable», le Conseil fédéral se garde bien de préciser qu'en réduisant aussi rapidement leur exposition aux risques de l'endettement international, les banques suisses ont contribué à l'échec de l'initiative Baker!

\section{Points marquants des analyses officielles}

Dans leurs analyses de la crise de l'endettement, les autorités fédérales ont insisté sur les points suivants:

- la situation souvent dramatique des pays surendettés s'explique à la fois par des causes internes et externes;

- le système financier international a résisté au choc;

- les banques suisses sont moins exposées aux risques;

- les débiteurs et les créanciers sont interdépendants, ils ont par conséquent des responsabilités communes;

- la crise ne peut pas être surmontée sans libéralisation économique et sans coopération internationale.

Certains traits de l'analyse ont évolué:

- le caractère structurel des problèmes de l'endettement ressort maintenant davantage;

- l'apparition sur le plan Nord-Sud de transferts financiers nets négatifs est déplorée (la réponse à l'interpellation Widmer ne le rappelle toutefois pas);

- les effets socio-économiques graves de la récession dans les pays surendettés et le manque de coopération internationale sont maintenant mieux reconnus;

- les autorités comprennent mieux l'importance du facteur temps.

\subsection{Comment surmonter la crise?}

Fondant son analyse sur le concept d'interdépendance mondiale et reconnaissant que les débiteurs et les créanciers partagent la responsabilité de la crise, le Conseil fédéral préconise une collaboration étroite entre toutes les parties concernées et une répartition équitable du coût des ajustements. II souhaite le renforcement du FMI en matière de surveillance économique. Bien que la Suisse n'en soit pas membre, elle se doit d'appuyer les institutions de Bretton Woods.

\section{Le rôle des créanciers}

L'effort des pays industrialisés consisterait à promouvoir une croissance sans inflation, à renoncer au protectionnisme, à ouvrir les marchés aux produits du Tiers Monde, à augmenter l'aide publique au développement et à stimuler les flux 
financiers privés destinés aux pays débiteurs. Dans sa lettre à l'Action Place financière suisse-Tiers Monde, le Conseiller fédéral Pierre Aubert a précisé ce dernier point: "Le secteur privé, en particulier bancaire, devrait se trouver en position de reprendre un rôle positif, en permettant un flux de ressources privées net sous forme de prêts et d'investissements vers les pays en développement, alors que le flux actuel est négatif.» (18)

Toujours selon la même lettre, il n'y a pas d'autre issue à la crise de l'endettement: «Ce n'est en effet qu'en fournissant aux pays endettés la possibilité économique d'honorer leurs propres engagements qu'une solution durable et satisfaisante pourra être trouvée.» (19)

\section{Le rôle des débiteurs}

Pour les pays en développement, l'ajustement consiste, selon les autorités suisses, à rétablir les équilibres qui ont été rompus sur les plans externe et interne, à entreprendre des réformes de structures favorisant l'économie de marché et à rétablir la confiance des détenteurs de capitaux. Ces transformations favoriseront le retour des capitaux de fuite, les investissements directs étrangers et l'ouverture de nouveaux crédits. Sur ces points, les autorités suisses sont d'accord avec le FMI. Elles défendent aussi le principe de la "conditionnalitén.

Cependant, au moment de l'adhésion de la Suisse aux Accords généraux d'emprunts, le Conseil fédéral a, conformément à une promesse faite au Conseil national, rappelé les principes de la législation suisse en matière de coopération au développement. La Suisse désire que ales programmes d'ajustement régulièrement requis par le FMI déploient des effets favorables à la réalisation des buts précités. Cela implique notamment que ces programmes permettent l'entretien et le développement de l'appareil de production à long terme et que les couches les plus défavorisées de la population n'aient pas à supporter une part excessive de l'ajustement.» (20)

En 1986, le Conseiller fédéral P. Aubert a écrit qu'aux yeux des autorités suisses, la politique du FMl était conforme à ce souhait (21). Ce satisfecit a toutefois peut-être été donné un peu hâtivement. En effet, à la Vile session plénière de la CNUCED, en juillet 1987, la délégation suisse a déclaré au Comité chargé d'examiner les problèmes des pays les moins avancés: la Suisse "voit dans une perspective nouvelle la nécessité et les formes de la conditionnalité...». Après avoir souligné l'utilité du dialogue, qui permet «de mieux comprendre la réalité et les besoins „ des bénéficiaires, d'en respecter les priorités et de soutenir les initiatives locales, la délégation suisse a poursuivi: «L'énonciation des modalités auxquelles l'aide extérieure continuera à être fournie ne doit intervenir que lorsque le gouvernement concerné a fixé sa politique et a intégré dans sa propre stratégie les suggestions des institutions financières internationales. Appliquée ainsi, la conditionnalité sert avant tout à garantir l'efficacité de l'assistance extérieure et par là son maintien, pendant plusieurs années, aux niveaux indispensables à la réussite des programmes de redressement.» (22) 


\section{Une stratégie double}

La Confédération préconise le traitement différencié des débiteurs (par catégories de pays et cas par cas), conformément aux recommandations du FMI, de la Banque mondiale, de l'OCDE, ainsi que du Sommet des sept plus grands pays industrialisés (qui s'est réuni dernièrement à Venise, en juin 1987). Au niveau du Tiers Monde, elle distingue donc la catégorie des pays à revenu moyen de celle des pays à revenu faible (dont font notamment partie les pays dits «les plus pauvres" ou "les moins avancés"). La première catégorie comprend la plupart des grands débiteurs, notamment ceux d'Amérique latine; dans la seconde, on retrouve la majorité des pays d'Afrique sub-saharienne. La réponse du Conseil fédéral du 16 septembre 1987 à l'interpellation Widmer contient quelques indications sur sa politique face à ces deux grandes catégories de débiteurs. (23)

Pour la première catégorie de pays, «il n'existe actuellement pas d'autre solution que celle poursuivie jusqu'ici, et qui consiste en une étroite collaboration entre créanciers et débiteurs. Les pays débiteurs doivent réaliser des programmes d'ajustement axés sur la croissance, lesquels favorisent l'épargne nationale et les investissements dans le pays et qui créent à l'étranger la confiance nécessaire à l'octroi de nouveaux crédits, tant officiels que privés. De leur côté, il faut que les pays industrialisés ne relâchent pas leurs efforts en vue de parvenir à une croissance économique durable et saine ainsi que d'ouvrir leurs marchés aux produits des pays débiteurs.» (23)

Ces recommandations font abstraction, semble-t-il, d'obstacles pourtant connus: les vives tensions entre certains débiteurs (par exemple le Brésil) et leurs créanciers, l'insuccès de l'initiative Baker (dû aux réticences des banques notamment), le manque de vigueur de la croissance dans les pays industrialisés, la remontée du protectionnisme, etc. Rappelons que les institutions multilatérales estiment qu'il faudrait, au contraire, prendre de nouvelles initiatives pour surmonter la crise de l'endettement. La Banque mondiale, par exemple, a écrit récemment que les conditions pour une croissance future n'étant pas encore réunies, il était probable que «laissé à lui-même, le marché ne procurerait pas le niveau de financement indispensable aux pays débiteurs engagés dans des programmes d'ajustement axés sur la croissance. De nouvelles initiatives sont requises dans l'intérêt à long terme des créanciers eux-mêmes, de même que des débiteurs et du système financier international dans son ensemble.» (24)

Concernant la seconde catégorie de pays (c'est-à-dire les pays à bas revenu, particulièrement en Afrique au sud du Sahara), le Conseil fédéral affirme qu'il sera en revanche nécessaire de développer des solutions qui allègent de manière substantielle leur dette.» (23)

De telles solutions sont actuellement à l'étude dans les organisations multilatérales et au Club de Paris. Elles visent principalement les pays les plus pauvres qui forment la majorité des pays à faible revenu, mais dont l'endettement exprimé en chiffres absolus est bas. Les pays dont les efforts d'ajustement seront jugés suffisants pourraient bénéficier d'un traitement spécial comportant des périodes de carence étendues, des délais de remboursement très longs, des taux d'intérêts 
réduits et, peut-être, des remises de dettes par les créanciers officiels. Ils seront aussi davantage soutenus par le FMl et par la Banque mondiale.

Le Club de Paris a déjà accordé de longs délais de remboursement à quelques pays, mais ses membres ne se sont pas encore entendus sur d'autres solutions. Ils pourraient bientôt annoncer quelques réductions d'intérêt sur des créances publiques. Par contre, il n'est pas question, semble-t-il, de mettre à contribution le secteur privé.

Le coût des mesures envisagées sera de toute façon bas. La dette extérieure qui écrase les pays qui en bénéficieraient représente seulement une petite fraction des créances détenues par les pays industrialisés. Par exemple, la dette publique et privée à long terme des pays africains à faible revenu atteignait 41 milliards de dollars à fin 1986 . Cette somme représentait seulement $5 \%$ du montant correspondant dû par l'ensemble du Tiers Monde ( 775 milliards). Quant au service de la dette estimé à 2 milliards de dollars, il représentait à peine $2 \%$ du service dû par le Tiers Monde (101 milliards). (25)

Ces chiffres expliquent en partie pourquoi le débat concernant les allègements de dette tend à se limiter au cas des pays les plus pauvres. Ils ont aussi le mérite de montrer qu'au niveau des négociations, les blocages ne sont pas de nature économique, mais politique.

\section{Interventions de la Confédération suisse}

L'action de la Confédération concernant l'endettement du Tiers Monde découle simultanément de la politique économique extérieure et de la coopération au développement (26). La première se préoccupe avant tout de la position de la Suisse dans l'ensemble des relations économiques internationales et de la défense de ses intérêts; la seconde "définit la contribution spécifique que peut apporter notre pays au progrès économique et social des pays en développement et à leur participation à l'économie mondiale, que ce soit directement par des mesures spéciales en faveur de ces pays ou indirectement par l'aménagement des relations économiques en général.» (27)

Ce chapitre traite de trois types d'interventions: des consolidations de dettes, de l'aide à la balance des paiements et des mesures qui tendraient à favoriser l'ajustement des flux financiers et commerciaux aux problèmes de l'endettement du Tiers Monde. Alors que le premier et le troisième types d'interventions sont essentiellement régis par les principes de la politique économique extérieure, le second est un instrument économique créé dans le cadre de la coopération au développement.

\subsection{Les consolidations de dettes}

Les premiers accords de consolidation de dettes conclus par la Confédération sont ceux de 1963 avec l'Argentine et de 1964 avec le Brésil. Ces accords ont fait l'objet 
de Messages aux Chambres. Pour pouvoir agir plus rapidement, le gouvernement a obtenu dès 1966 une délégation de pouvoirs; celle-ci a été prorogée en 1970 et en 1980 (28). Il est actuellement autorisé à consolider des créances appartenant à la Confédération ou couvertes par la Garantie contre les risques à l'exportation (GRE).

\section{Négociations multilatérales et bilatérales}

Les pourparlers en vue d'une consolidation de dettes commerciales garanties se déroulent normalement en deux temps: sur le plan multilatéral d'abord (au Club de Paris), sur le plan bilatéral ensuite.

Au cours de la première phase de négociations, le pays débiteur informe les créanciers de sa situation économique, annonce la politique de redressement qu'il a convenue avec le FMI (29) et formule la demande de rééchelonnement. Les institutions internationales et les pays représentés à la Conférence présentent à leur tour leurs analyses et leurs propositions. Les participants discutent ensuite des modalités du rééchelonnement: la nature des dettes consolidées, la période couverte par l'accord, la durée du rééchelonnement, le délai de grâce, etc. A l'issue de la conférence, les participants signent un "procès-verbal agréé" qui n'a pas force légale, mais qui a valeur de recommandation en vue des négociations bilatérales.

Seuls les pays dont les créances atteignent un montant minimal d'un demimillion de DTS (ou parfois davantage) sont invités à signer le uprocès-verbal agréé". Les autres devraient être remboursés selon les échéanciers contractuels ou, pour les paiements en retard, dans les meilleurs délais.

La seconde phase de négociations se déroule au niveau bilatéral. Le taux d'intérêt sur les capitaux consolidés est fixé à ce moment, aux conditions du marché. Le compromis atteint à Paris doit être respecté. Si le pays débiteur faisait des concessions supplémentaires à l'un des créanciers, les autres pourraient exiger d'en bénéficier également, au nom du principe de la non-discrimination.

\section{Consolidation des créances suisses}

En 1986, 17 accords-cadre ont été conclus au Club de Paris. La Suisse en a signé 13.

Les exportateurs doivent accepter la consolidation de leurs créances (30). La GRE calcule toutefois les indemnités sur la base de l'échéancier contractuel, en fonction du degré de couverture. Plus tard, lorsque les pays débiteurs paient des intérêts ou des amortissements dans le cadre des accords de consolidation, les exportateurs et la GRE se partagent les montants encaissés, toujours en fonction du taux de couverture (31).

A fin décembre 1986, 50 accords étaient en vigueur entre la Suisse et 24 pays (y compris quelques pays de l'Est). L'avoir de la GRE résultant de ces accords 
totalisait 1,1 milliard de francs (32). De leur côté, les exportateurs détenaient un peu plus de 400 millions de francs en créances consolidées (33).

\section{Mesures d'allègement du service de la dette}

Avec l'aggravation de la crise de l'endettement dans certains pays du Tiers Monde, les instruments classiques ne suffisent plus. Aussi le Club de Paris a-t-il récemment fait quelques innovations. Signalons notamment l'introduction encore timide d'accords multiannuels de rééchelonnement de dettes et l'extension des délais de remboursement.

Dans le cadre des accords multiannuels, la Suisse s'était entendue avec la Côte d'lvoire sur le rééchelonnement des créances venant à échéance en 1986, 1987 et 1988.

L'accord du 28 janvier 1987 prévoyait qu'il serait exécuté par phases successives à condition que la Côte d'Ivoire applique le programme économique élaboré en consultation avec le FMI. Subissant une nouvelle chute importante de ses recettes d'exportation, la Côte d'Ivoire a obtenu l'abrogation de cet accord. De nouvelles négociations sont prévues.

L'extension des délais de remboursement s'étend à 15 ans (avec une période d'attente de 6 ans) et, dans le cas de certains pays très pauvres, jusqu'à 20 ans (avec 10 ans d'attente) (34). La Suisse détient peu de créances sur les pays qui ont commencé à bénéficier de ces mesures. Aussi n'avait-elle été associée, jusqu'en septembre 1987, qu'à un seul accord multilatéral prévoyant de tels délais. Le bénéficiaire en est le Zaïre qui a obtenu des délais de 15 ans.

Dans de nombreux cas, comme par exemple dans celui du Mozambique en juin 1987, l'extension des délais n'entraîne toutefois qu'un report du constat d'insolvabilité. A moins qu'un miracle ne se produise dans quelques années, de telles dettes ne seront jamais remboursées. La valeur réelle des créances est donc pratiquement nulle. Pourquoi repousse-t-on alors l'heure de vérité? Des annulations de dettes ne clarifieraient-elles pas la situation?

N'oublions pas que l'extension de la période de grâce et du délai de remboursement affecte seulement le principal. Le débiteur doit continuer à s'acquitter des intérêts. Le créancier qui désire investir ailleurs peut se refinancer sur les marchés des capitaux. Précisons encore que dans le cas des pays les plus défavorisés, les appuis consentis par les donneurs bilatéraux et multilatéraux permettent généralement aux débiteurs d'effectuer le paiement des intérêts, alors que des importations de biens essentiels sont souvent interrompues. Les créanciers s'accommodent donc des rééchelonnements de dettes, même lorsque les débiteurs bénéficient de délais très longs.

\section{Obstacles aux annulations de dettes}

Il existe aussi des obstacles inhérents aux structures spécifiques de chacun des pays membres du Club de Paris. En Suisse, on peut distinguer trois types d'obstacles: 
- La Confédération ne détient plus de créances sur les pays les plus pauvres. Or ceux-ci ont le plus de chances de bénéficier d'une éventuelle remise de créances publiques par les membres du Club de Paris. Al'occasion d'une action internationale décidée en 1977, la Confédération avait remis aux pays les plus pauvres des créances s'élevant à quelque 80 millions de francs. Depuis, les financements publics accordés à ces pays l'ont été sous la forme de dons (35).

- A fin 1986, les avoirs de la GRE résultant d'accords de consolidation de dettes s'élevaient, comme je l'ai déjà mentionné plus haut, à 1,1 milliard de francs. Les créances sur des pays à faible revenu atteignaient près de 260 millions (23\% du total). Juridiquement indépendante depuis 1981, la GRE doit tenter d'équilibrer ses comptes. La Confédération a néanmoins dû lui avancer des sommes considérables (presque 1 milliard a fin 1986) sur lesquelles un intérêt est calculé au taux moyen des emprunts fédéraux. Si elle acceptait l'annulation de créances détenues par la GRE, la Confédération devrait certainement renoncer à son tour au remboursement d'une partie de ses avances.

- Quant aux créances consolidées détenues par les exportateurs, elles s'élevaient à 400 millions de francs à fin 1986. Une centaine de millions concernait des pays à faible revenu. Rappelons que ces sommes correspondent aux parts du risque non couvertes par la GRE. Les exportateurs sont tenus de participer à des consolidations (aussi lorsqu'elles comportent des délais étendus), mais pas à des annulations de dettes.

Ces obstacles expliquent dans une bonne mesure l'opposition des autorités fédérales aux annulations de dettes commerciales. Peut-on néanmoins s'attendre à un geste en faveur des pays les plus déshérités? Le Conseil fédéral ne s'est pas prononcé sur ce point. En septembre 1987, il a éludé la question du Conseiller national S. Widmer: «Envisage-t-on en Suisse une remise totale de la dette des pays les plus pauvres (6\% de la dette totale)? " (36). II paraît toutefois certain que la Suisse ne fera aucun geste dans ce domaine en l'absence d'une action commune des membres du Club de Paris.

En ce qui concerne les créanciers privés, on comprend qu'ils préfèrent l'extension des délais de remboursement à des annulations de dettes. A première vue, cette politique parait d'ailleurs aussi préférable pour la GRE. Qu'en est-il réellement? Pour comprendre l'enjeu, il faut considérer les principales causes du déséquilibre des comptes de la GRE:

- Les pertes de la garantie contre les risques de change s'élèvent à environ 600 millions de francs. Le Conseil fédéral a suspendu l'octroi de cette garantie le 1 er avril 1985 pour éviter des pertes supplémentaires.

- A fin 1986, la GRE a dú constituer une provision pour créances douteuses de 340 millions de francs. (Ce montant représente 30\% des 1114 millions de créances consolidées nettes.)

Il faut bien distinguer ces deux causes. La première concerne des pertes irrémédiables, mais dont le montant est connu. La seconde correspond à l'estimation 
d'un risque: le montant des pertes effectives dépendra de l'évolution de la crise de l'endettement. Aujourd'hui, les autorités fédérales sont encore convaincues que le refus des annulations de dettes peut limiter efficacement les pertes des créanciers. A la longue, ce raisonnement pourrait bien se révéler faux.

Le Conseiller fédéral Kurt Furgler avait déclaré en mars 1986, à propos des pertes subies dans le cadre de la garantie contre les risques monétaires, que "l'heure de vérité arriverait tôt ou tard" (37). Ces paroles s'appliquent aussi à la garantie contre les risques politiques. Si la Confédération décidait un jour d'abandonner une partie des montants qu'elle a avancés, dans le but de remédier à la première cause du déséquilibre des comptes de la GRE, pourquoi ne consentirait-elle pas, également dans le cadre de la politique économique extérieure, un effort similaire en faveur des pays surendettés? (38)

II resterait finalement à trouver un arrangement équitable avec les exportateurs. La Confédération ne peut pas les priver de leurs droits, mais elle n'est pas non plus tenue, pour défendre ces droits, d'exercer sur les pays débiteurs des pressions incompatibles avec les objectifs de la coopération au développement. Une solution mutuellement acceptable ne consisterait-elle pas dans le rachat, par la Confédération, des créances à un cours réaliste?

\section{Vers des taux d'intérêt réduits?}

Lors des consolidations de dettes, les pays membres du Club de Paris exigent des taux d'intérêt adaptés aux conditions usuelles des marchés financiers. Plus précisément, ces taux couvrent les coûts de refinancement, les frais administratifs et les risques supportés par les créanciers. Les coûts de refinancement des créances consolidées, qui représentent la charge la plus importante, varient selon l'évolution des taux d'intérêt sur les marchés financiers nationaux et internationaux. Les différents créanciers (agences de garantie des risques à l'exportation, banques commerciales et exportateurs) se refinancent selon des modalités spécifiques et à des coûts différents. Les accords prévoient des taux d'intérêt valables pour toute la durée des consolidations.

Les accords bilatéraux conclus par la Confédération prévoient des paiements en francs suisses. Les taux d'intérêt ont été fixés à $7 \%$ en 1985 , à 6 3/4\% en 1986 et à $6 \%$ en 1987. Les coûts de refinancement à la charge des créanciers ont en effet sensiblement baissé. Le calcul des taux d'intérêt semble

avoir reposé sur une appréciation assez large des coûts de refinancement, des frais administratifs et des risques.

La Confédération envisage seulement des réductions des taux d'intérêt en faveur des pays les plus pauvres, et $c e$ avec beaucoup de réticences. Dans sa réponse du 16 septembre 1987 à l'interpellation Widmer, le Conseil fédéral délimite le cercle des bénéficiaires, définit la conditionnalité d'une mesure éventuelle et attire l'attention sur certaines difficultés juridiques:

- les bénéficiaires seraient ades pays en développement les plus pauvres et les plus endettés" (donc essentiellement d'Afrique sub-saharienne); 
- il s'agira de pays qui «sont disposés à entreprendre une réforme prometteuse de leur économien;

- «il conviendra évidemment de tenir dûment compte des particularités institutionnelles et financières de notre GRE». (39)

Le dernier argument soulève un problème qui se poserait surtout si la mesure envisagée devait bénéficier à des débiteurs importants. Vu le montant modeste, en chiffres absolus, des créances de la GRE sur les pays les plus pauvres, la réduction d'intérêts envisagée par le Conseil fédéral aurait peu d'incidences financières.

Dans une interview récente, F. Blankart, Directeur de l'Office fédéral des affaires économiques extérieures, a insisté sur une des difficultés juridiques: l'abaissement des taux d'intérêt serait-il compatible avec le respect des droits des exportateurs (et de leurs banques)? (40) Ceux-ci restent propriétaires de la part des créances garanties non couvertes par la GRE. Espérons que les trois éléments suivants seront dûment pris en considération dans l'analyse de ce problème: la faible valeur réelle de certaines créances, l'asymétrie des effets d'une réduction des taux d'intérêts pour les débiteurs et pour les créanciers, et enfin l'acceptation par ces derniers d'assumer le solde du risque.

Dans le débat sur des réductions éventuelles des taux d'intérêt, les considérations juridiques et financières liées à des particularités institutionnelles occupent une grande place. N'oublions pas un élément essentiel du problème: la reconnaissance, par la Confédération, des responsabilités de la Suisse dans la crise de l'endettement (41). La Confédération suisse a par conséquent l'obligation de s'attaquer résolument aux problèmes restés en suspens dans la cadre traditionnel des consolidations de dettes.

Deux types de mesures paraissent aujourd'hui indispensables:

- Inclure uniquement les coûts de refinancement dans le calcul des taux d'intérêt lors des consolidations de dettes. Les débiteurs n'auraient donc plus à supporter les frais administratifs des créanciers et à financer la couverture des risques. Ces charges seraient comprises une fois pour toutes dans le calcul des émoluments facturés par la GRE (pour la part des créances qu'elle accepte de couvrir) ou seraient supportées par les banques et par les exportateurs (pour la part non couverte par la GRE).

- Accorder des réductions supplémentaires aux pays à faible revenu surendettés. Ceux qui resteront probablement longtemps insolvables devraient bénéficier de l'exemption totale des intérêts (ou d'annulations de dettes). L'efficacité de ces mesures pourrait être améliorée grâce à la coordination avec d'autres interventions de la Confédération, en particulier dans le cadre de la politique économique extérieure et de la coopération au développement.

\section{Susciter un consensus favorable}

Pour obtenir l'appui du Parlement et des citoyens, la Confédération devrait revoir sa politique de l 'information. La délégation de pouvoirs accordée par les Chambre 
en 1966 et prorogée à deux reprises depuis dispense le Conseil fédéral de leur soumettre chaque consolidation de dettes. Ce changement était judicieux car il faut pouvoir agir rapidement. En contre partie, le Conseil fédéral a l'obligation de donner une information périodique (42). Les renseignements fournis annuellement dans le Rapport sur la politique économique extérieure sont malheureusement insuffisants. En janvier dernier, par exemple, ce rapport indiquait seulement les montants des sept consolidations de dettes accordées à des pays du Tiers Monde en 1986 (43)! Quant au Rapport annuel de la GRE, il s'est étoffé depuis quelques années, mais l'information sur les consolidations de dettes reste encore très limitée.

\subsection{L'aide à la balance des palements (ABP) (44)}

"L'ABP qui complète l'aide au titre de projets vise à utiliser les capacités (de production) existantes en finançant les importations dans des secteurs économiques importants"; elle permet également aux pays bénéficiaires "de supporter des difficultés d'approvisionnement, tandis que l'ajustement structurel de l'économie peut être entrepris" (45).

\section{Un instrument de la coopération au développement}

Comme les autres formes de coopération publique, l'ABP est subordonnée à la loi fédérale sur la coopération au développement de 1976. Selon les buts définis à l'article 5 de la loi, la coopération suisse au développement vise à soutenir les efforts des pays en développement en vue d'améliorer les conditions de vie de leur population, en particulier des groupes les plus défavorisés, à contribuer à mettre ces pays en mesure d'assurer leur développement par leurs propres forces et à tendre, à long terme, vers un meilleur équilibre au sein de la communauté internationale.

L'ABP «est destinée en premier lieu aux pays en développement pauvres, pour lesquels un ajustement entraînerait normalement de lourdes pertes, tant du point de vue de la production et de l'emploi que du revenu. Et plus la période est courte, plus les répercussions de cette adaptation sont négatives. Notre aide s'étale donc sur plusieurs années et revêt habituellement la forme de contributions non remboursables" (45).

L'aide bilatérale de la Suisse s'inscrit dans le cadre d'une "action coordonnée à l'échelle internationale. Cela revient à dire qu'en règle générale un cercle de bailleurs de fonds au titre de mesures bilatérales ou multilatérales soutient un Etat du Tiers-Monde dans les efforts qu'il entreprend pour assainir et restructurer son économie... Un tel soutien international a fréquemment pour point de départ une consolidation de dettes multilatérale, un programme de stabilisation ou d'ajustement structurel avec le concours d'une organisation internationale ou bien une action ad hoc d'un groupe consultatif» (45). Pour le Conseil fédéral, la 
coordination des actions sur le plan international est indispensable.

La Confédération accorde les ABP par le truchement de l'aide bilatérale ou dans le cadre de cofinancements (46):

- Dans le cas des aides bilatérales, l'affectation des fonds est fixée d'entente entre le pays destinataire et la Confédération. Cette solution prévaut lorsque la Suisse entretient déjà des relations régulières avec ce pays que ce soit dans le domaine de nos exportations (concernant des secteurs prioritaires du programme d'ajustement structurel) ou dans celui de la coopération technique.

- Dans le cas des cofinancements, les fonds suisses sont affectés aux programmes d'ajustement structurel soutenus par l'IDA. Cette formule prévaut surtout lorsque la présence suisse est moins active que dans le premier cas.

\section{Insertion dans la politique économique extérieure}

Le Conseil fédéral indique qu'il «est dans notre intérêt de sauvegarder la stabilité économique d'un pays en développement pour maintenir nos relations avec lui. Les perturbations de la balance des paiements entrainent des restrictions à l'importation et l'insolvabilité, ce qui se répercute négativement sur l'ensemble de notre économie ou certaines de ses branches.» (45)

Les fonds accordés au titre de l'ABP ne sont généralement pas liés à des livraisons suisses. «Cependant, vu que nombre de pays qui bénéficient d'une $A B P$ de la Suisse entretiennent avec notre pays des relations commerciales traditionnelles relativement importantes dans des secteurs prioritaires, - leurs industries sont équipées de machines suisses -, une grande partie des fonds bilatéraux a été utilisée à ce jour pour acheter des biens fabriqués en Suisse.» (46)

\section{Quelques chiffres (47)}

Entre 1980 et 1986, la Confédération a accordé une somme totale de quelque 178 millions de francs au titre de l'ABP (48). Quinze accords ont été conclus avec neuf pays: le Bangladesh (accord de 1980), le Soudan (1981, 1983 et 1984), Madagascar (1982, 1984 et 1985), le Ghana (1984, 1985 et 1986), la Guinée-Bissau (1984), la Zambie (1985), le Sénégal, la Guinée et la Bolivie (1986). A l'exception du Bangladesh et de la Bolivie, tous les destinataires sont africains. Il était prévu qu'un accord serait également conclu avec Haïti (automne 1987).

Dans neuf cas, les modalités de l'aide ont été négociées directement avec le pays concerné; dans les sept autres cas, la Suisse a soutenu des programmes administrés par l'IDA (les cofinancements font aussi l'objet d'un accord entre la Suisse et les pays bénéficiaires).

Les contributions suisses ont servi à financer les importations courantes de certaines branches économiques des pays destinataires. Dans le cas des neuf contributions bilatérales, $80 \%$ en moyenne des biens ont été livrés par des en- 
treprises suisses. Les commandes ont notamment bénéficié à l'industrie chimique (colorants, pesticides, herbicides, médicaments), ainsi qu'à l'industrie des machines textiles (pièces de rechange et composants).

La Confédération a décidé de renforcer l'ABP. Elle prévoit de lui consacrer 120 millions de francs entre 1987 et 1989 . Ces moyens devraient permettre de conclure 10 ou 12 nouveaux accords.

\section{Remarques}

L'ABP est un instrument récent de la coopération au développement qui permet de procurer rapidement des devises à des pays confrontés à une crise aiguë de la balance des paiements. Subsidiairement, il sert aussi très bien la politique économique extérieure.

Cette convergence d'objectifs n'est pas gênante en soi. Elle peut même renforcer l'efficacité des interventions. Tant que les buts de la coopération au développement resteront déterminants, on pourra renforcer l'ABP sans hésitation. Avec 40 millions de francs par an, la Confédération ne peut pas mener des actions d'une envergure suffisante, même en limitant strictement le cercle des bénéficiaires.

Cependant, le renforcement des ABP (suisses et étrangères) devrait concorder avec des progrès réels dans le domaine des allègements de dettes. Sinon les ressources ordinaires en devises (par ex. les recettes d'exportation) libérées grâce à l'octroi de l'ABP seront affectées au service de la dette et non à la réalisation des programmes de développement.

\subsection{L'ajustement des flux financiers et commerciaux}

Le Conseil fédéral reconnaît que les débiteurs et les créanciers partagent la responsabilité de la crise de l'endettement. Des ajustements doivent être faits au Nord comme au Sud. Malheureusement les textes officiels ne disent pratiquement rien sur les ajustements qui incombent à la Suisse.

Quelles adaptations de son économie la Suisse devrait-elle entreprendre pour promouvoir des relations équitables avec le Tiers Monde et une issue favorable à la crise de l'endettement? Cette question étant vaste, j'aborderai seulement quelques points concernant les flux financiers et commerciaux.

\section{Déséquilibres des flux financiers}

La Suisse bénéficie d'un excédent structurel de la balance des opérations courantes. En 1986, le solde actif s'est élevé à 12 milliards de francs. Ce montant a presque atteint $5 \%$ du produit national brut. Comme les années précédentes, on a observé un déficit de la balance commerciale, mais de larges excédents au 


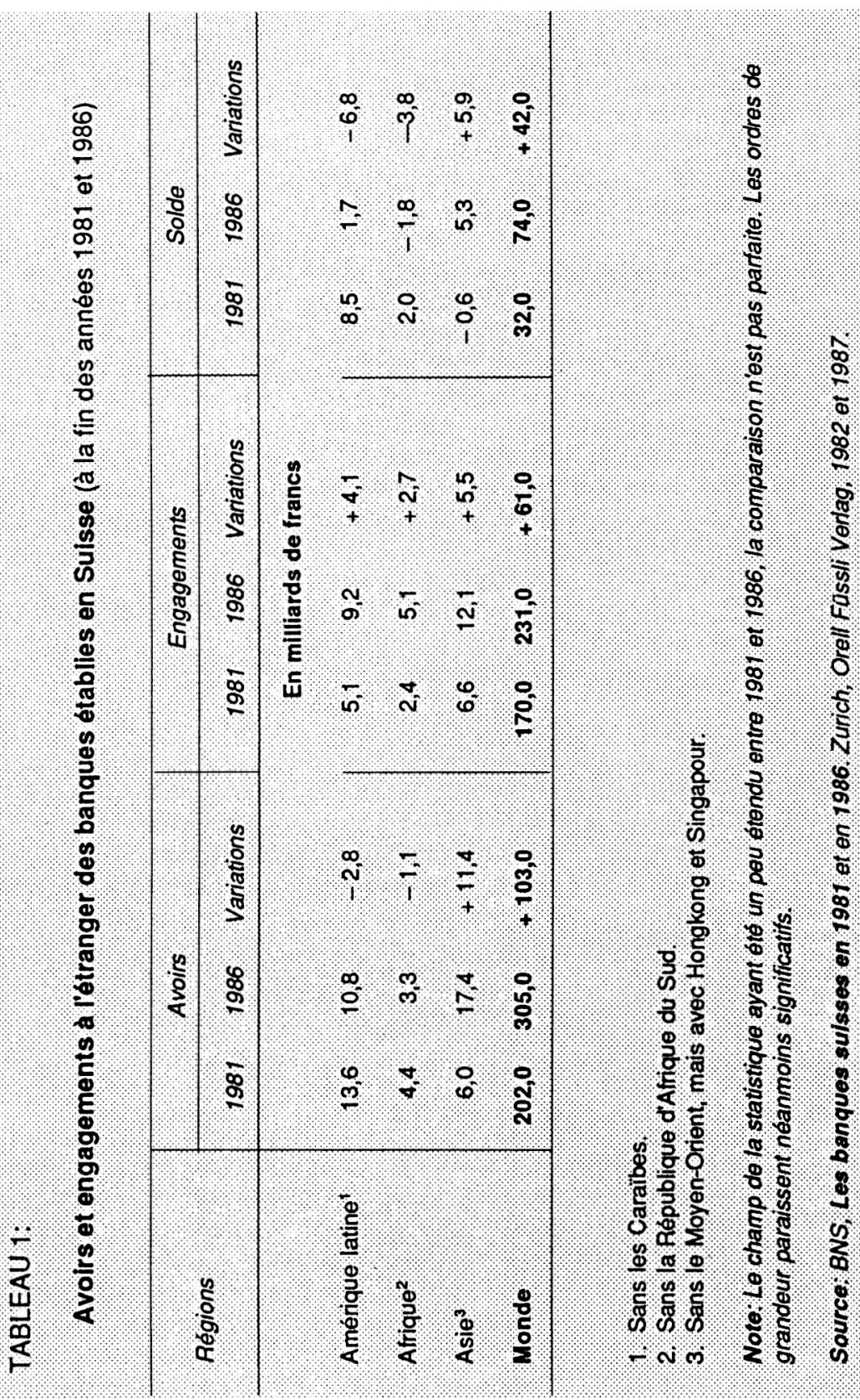


niveau des services et des revenus de capitaux. L'excédent structurel de la balance des opérations courantes confère à la Suisse une forte capacité de financement extérieur. En 1986, ce sont les banques commerciales qui ont réalisé les exportations nettes de capitaux les plus grandes (10 millions de francs) (49).

L'absence de ventilation géographique des montants figurant dans la balance des paiements limite les possibilités d'analyse. Contrairement aux relations avec les pays industrialisés, les échanges commerciaux de la Suisse avec le Tiers Monde sont largement excédentaires (6 milliards de francs en 1986). Quant àl 'excédent de la balance des opérations courantes, il est certainement considérable. Il dépasse probablement, en proportion, celui des opérations courantes effectuées avec les pays industrialisés. Enfin, alors que les places financières occidentales et asiatiques voient affluer les capitaux en provenance de la Suisse, les régions surendettées du Tiers Monde subissent le phénomène inverse. L'étude des bilans bancaires montre clairement ce phénomène (cf. tableau $N^{\circ} 1$ ).

Les banques ont retiré une bonne partie de leurs avoirs d'Amérique latine et d'Afrique, malgré la progression rapide de leurs placements dans le monde. Elles ont en outre bénéficié de l'afflux de capitaux en provenance de ces régions. La position nette (avoirs moins engagements) des banques suisses y a par conséquent fortement régressé. Par rapport à l'Afrique, elle est devenue négative. Si la tendance observée depuis l'éclatement de la crise de l'endettement devait se poursuivre, il en irait bientôt de même par rapport à l'Amérique latine!

\section{Déséquilibres des flux commerciaux}

Alors que la balance commerciale de la Suisse est traditionnellement déficitaire, les échanges avec le Tiers Monde se soldent, comme nous l'avons constaté, par un excédent considérable (50). Le tableau 2 montre la répartition par groupes de pays.

Le coefficient d'échanges représente le meilleur indicateur concernant les déséquilibres des flux commerciaux. En 1986, les exportations ont représenté plus du double des importations pour presque tous les groupes de pays qui figurent au tableau.

\section{Manque de financements pour l'Afrique et pour l'Amérique latine}

C'est avec l'Asie que la Suisse réalise l'excédent commercial le plus élevé, mais ce continent bénéficie aussi de la progression des flux financiers en provenance de notre pays. En revanche, l'Afrique et l'Amérique latine enregistrent simultanément, dans leurs échanges avec la Suisse, des mouvements nets de capitaux privés négatifs et des déficits commerciaux!

La comparaison entre les tableaux 2 et 3 révèle, en ce qui concerne l'Afrique et l'Amérique latine, une contradiction flagrante entre le comportement des banques suisses, qui est extrêmement prudent, et celui des entreprises industrielles qui ont toujours besoin de ces débouchés. Les pays d'Afrique et d'Amérique 


\section{TABLEAU 2:}

Commerce de la Suisse avec le Tlers Monde en 1986 Importations, exportations, solde de la balance commerciale

\begin{tabular}{|c|c|c|c|c|c|c|}
\hline \multirow[t]{2}{*}{ Groupes de pays } & \multicolumn{2}{|c|}{ Importations } & \multicolumn{2}{|c|}{ Exportations } & \multirow{2}{*}{$\begin{array}{l}\text { Solda } \\
\text { Mio Fr }\end{array}$} & \multirow{2}{*}{$\begin{array}{l}\text { Coefficient } \\
\text { d'échange } \\
\text { Exp./Imp. }\end{array}$} \\
\hline & Mio F & $\%$ & Mio Fr. & $\%^{\prime}$ & & \\
\hline \multicolumn{7}{|l|}{ Ensemble des pays } \\
\hline $\begin{array}{l}\text { du Tiers Monde } \\
\text { a.Groupes } \\
\text { géooranhiques: }\end{array}$ & 4.837 & 6,58 & 10.786 & 16,10 & +5.949 & 2,23 \\
\hline Afrigue & 1.088 & 1,48 & 1.552 & 2,32 & +464 & 1,43 \\
\hline Amérique & 1.116 & 1,52 & 2.291 & 3,42 & +1.175 & 2,05 \\
\hline Asio & 2.624 & 3,57 & 6.908 & 10,31 & +4.284 & 2,63 \\
\hline \multicolumn{7}{|l|}{ b.Groupes } \\
\hline \multicolumn{7}{|l|}{ économiques: } \\
\hline $\begin{array}{l}\text { Exportateurs de } \\
\text { pétrole }\end{array}$ & 1.197 & 1,63 & 3.706 & 5,53 & +2.590 & 3,10 \\
\hline $\begin{array}{l}\text { Exportateurs } \\
\text { d'articles manu- }\end{array}$ & & & & & & \\
\hline facturés & 1.719 & 2,34 & 3.043 & 4,54 & +1324 & 1,77 \\
\hline Autres pays & 1.920 & 2,61 & 4.036 & 6.02 & +2116 & 2,10 \\
\hline
\end{tabular}

1. Part du commerce extérieur de la Suisse.

Note: La classification est expliquée dans la partie statistique de l'Annualre. Le tableau a été réalisé par P.G. Cancellieri.

Source: Statistique annuelle du commerce extérieur de la Suisse 1986, Vol. I. Berne, Direction générale des douanes, 1987, pp. 542-544.

latine doivent par conséquent souvent financer leurs importations en provenance de la Suisse par des devises gagnées ou empruntées ailleurs!

Il apparaît donc que la Confédération devrait vivement encourager la reprise des crédits aux pays d'Afrique et d'Amérique latine, dans la mesure où ces financements seront destinés à des projets nécessaires au développement. Simultanément, elle devrait chercher à corriger les déséquilibres excessifs au niveau des relations commerciales. Considérons d'abord les exportations, puis les importations. 


\section{TABLEAU 3:}

\section{Mouvements nets de capitaux privés entre la Suisse, I'Afrique et l'Amérlque latine, en 1986}

\begin{tabular}{|c|c|c|}
\hline & Afriquo & Amérique latino \\
\hline & \multicolumn{2}{|c|}{ En mio de fr. } \\
\hline Crédits a lexportation garantis & -286 & -99 \\
\hline Investissements directs & +54 & +418 \\
\hline $\begin{array}{l}\text { Institutions régionales de } \\
\text { financement du développement }\end{array}$ & +100 & +269 \\
\hline Flux bancaires & $=490$ & $=1319$ \\
\hline Total & -622 & -731 \\
\hline
\end{tabular}

Note La Banque mondiale a effectué en 1986 des emprunts nets de 377 millions do francs en Suisse. Une partie de cette somme a aussie été prétée aux pays africains et latino-américains.

Source. DDA et OFAEE, Mémorandum de la Sulsse au Comlté d'alde au développement 1986. Berne, 1987, chap. XVI "Les flux prives".

\section{Ajuster les flux d'exportations}

La Confédération peut agir dans une certaine mesure sur l'évolution des exportations. La garantie contre les risques à l'exportation (GRE) exerce une influence non négligeable, même si elle n'en couvre aujourd'hui plus qu'une petite partie. La GRE a pour but de développer les possibilités de travail en Suisse et de promouvoir le commerce extérieur. Cependant, «s'agissant d'exportations à destination des pays en développement les plus défavorisés, la Confédération tiendra compte des principes fondamentaux de la politique suisse en matière d'aide au développement». (51)

En principe, cette disposition tend donc à décourager des exportations défavorables au développement des pays emprunteurs. Elle semble jouer particulièrement ce rôle lorsque les effets défavorables au développement augmentent le risque financier. Cependant, la GRE ne fournit pas d'informations qui permet- 
traient d'apprécier les incidences de la disposition sur le niveau des exportations suisses. Elle donne seulement la liste des pays auxquels la disposition s'applique. II s'agit d'une soixantaine de pays à faible revenu. Environ $20 \%$ des montants garantis par la GRE concernaient ce groupe de pays à fin 1986, alors que la part de l'ensemble des pays en développement s'élevait à $70 \%$. Pour les nouvelles garanties accordées pendant l'année 1986, ces parts étaient respectivement de $15 \%$ et de $75 \%$. Moins d'un tiers du montant des garanties concernant des exportations vers le Tiers Monde doivent donc faire l'objet d'une appréciation des effets sur le développement. C'est peu!

Par contre, lorsque des pays ont besoin de biens suisses dans le cadre de leur politique de développement, la disposition ne sert pas à faciliter l'octroi des garanties et, par conséquent, l'obtention d'un financement. Dans ces cas, la GRE s'en tient à l'analyse du risque financier. Maintenant que les pays surendettés n'obtiennent plus suffisamment de crédits, ne faudrait-il pas que la disposition concernant les principes fondamentaux de la politique de développement puisse aussi, dans des cas bien étudiés, favoriser l'octroi de garanties?

\section{Ajuster les flux d'importations}

Le Conseil fédéral est fréquemment interpellé sur ce point. En mars 1987, le Conseiller national S. Widmer avait demandé s'il était possible d'augmenter les importations en provenance des pays en développement grâce à l'assouplissement de certaines restrictions. Dans sa réponse, le Conseil fédéral affirme que le marché suisse est très ouvert aux produits industriels étrangers et moins fermé qu'on ne l'imagine généralement aux matières premières et aux denrées alimentaires. II compte sur les nouvelles négociations du GATT pour favoriser les exportations des pays en développement et rappelle que «la Suisse a pris de son côté des mesures autonomes dans le domaine des préférences tarifaires et de la promotion des ventes de produits importés des pays en développement». (52)

II est vrai que le régime douanier est ouvert aux importations de produits manufacturés et que les préférences tarifaires accordées par la Suisse aux pays en développement sont relativement favorables. Par contre, dans le domaine agricole, la Suisse est résolument protectionniste. D'autre part, on connaît mal les effets de la politique d'approvisionnement des entreprises privées sur les importations en provenance du Tiers Monde. Quant à la promotion des importations réalisée dans le cadre de la coopération au développement, elle est utile, mais ses moyens sont très insuffisants.

Le Conseil fédéral aura l'occasion de revenir sur ces questions dans ses réponses à l' ' Appel pour un commercé équitable avec le Tiers Monde déposé en octobre 1986 par sept œuvres d'entraide et au postulat Longet du 11 juin 1987 (53). En attendant, relevons une phrase significative dans le rapport de la commission des pétitions chargée d'examiner l'"Appel pour un commerce équitable avec le Tiers Monde»: "La commission a constaté que l'appel soulevait un problème 
complexe qui est périodiquement évoqué au Parlement... La commission regrette de ne pas connaître dans leurs grandes lignes les priorités et les intentions du Conseil fédéral en la matièren. (54)

\section{Ajustement en profondeur et actions concrètes}

La mise en place d'une politique d'ajustement des flux financiers et commerciaux de la Suisse prendra nécessairement du temps. Les résultats éventuels apparaîtront encore plus tard.

Vu la dégradation de la situation dans les pays surendettés et la persistance de la crise de l'économie mondiale, il faut des interventions d'urgence. Aussi la Confédération devrait-elle, au cours de la phase de définition d'une politique d'ajustement en profondeur, entreprendre rapidement quelques actions concrètes dans ce sens. Elle pourrait par exemple, à l'aide de taxes sur certaines transactions commerciales et financières, constituer un fonds destiné à compenser la baisse des cours des matières premières (55), soutenir la diversification des exportations des pays en développement, financier les aides à la balance des paiements et contribuer à des allègements de dettes.

\section{Conclusion}

Au terme de cet examen des analyses, des objectifs et des interventions de la Confédération, il convient d'apprécier dans quelle mesure nos autorités ont tiré des leçons de la crise de l'endettement et comment elles les ont traduites en actes (ou comment elles pourraient encore le faire).

Alors qu'en 1984, le Conseil fédéral avait encore tendance à considérer le problème de l'endettement comme un phénomène transitoire, dû à une « surcharge temporaire» (56) du système, aujourd'hui il en reconnaît clairement le caractère structurel. II constate que les programmes de redressement appliqués par les pays débiteurs n'ont pas obtenu les résultats attendus et que les pays créanciers n'ont ni apporté des appuis suffisants, ni procédé aux ajustement indispensables de leurs propres structures économiques.

Non-membre des institutions de Bretton-Woods, la Confédération s'en est néanmoins rapprochée grâce à l'adhésion aux Accords généraux d'emprunts et à l'octroi de cofinancements à l'IDA. Le Conseil fédéral a plusieurs fois indiqué son accord avec le principe de la conditionnalité appliqué aux pays débiteurs, mais il a aussi rappelé son attachement à deux principes fondamentaux de la coopération suisse: la priorité accordée aux groupes les plus défavorisés de la population et le droit des pays débiteurs d'intégrer les conditions des bailleurs de fonds dans leurs propres stratégies de développement.

Malgré la reconnaissance de la coresponsabilité des pays créanciers, les autorités fédérales n'ont pas effectué pour l'instant un repérage précis, en Suisse, des comportements économiques et des distorsions structurelles qui favorisent le surendettement des pays en développement. II s'agit certes d'une tâche difficile. 
Les documents officiels n'analysent pas, en particulier, les effets des déséquilibres des relations commerciales et financières de la Suisse avec le Tiers Monde.

En ce qui concerne la traduction en actes, au niveau des interventions de la Confédération, on observe quelques adaptations de la politique économique extérieure et le renforcement de la coopération au développement. Rappelons notamment la participation de la Suisse à des consolidations de dettes plus favorables aux débiteurs et quelques améliorations de la politique commerciale: la prise en considération, encore très timide certes, de la coopération au développement lorsque la GRE traite de dossiers concernant les pays à faibles revenus et la poursuite de l'extension du schéma suisse des préférences tarifaires. D'autre part, la Confédération a renforcé l'aide à la balance des paiements.

Cependant, considérées globalement, les interventions de la Confédération manifestent bien de la bonne volonté mais elles sont malheureusement sans commune mesure avec la gravité de la crise et avec la prospérité de la Suisse.

Les pressions et les appels en faveur d'un engagement à la hauteur de nos responsabilités se multiplient toutefois. Grâce aux leçons qu'elle a tirées de la crise, la Confédération pourrait notamment développer son action dans deux domaines essentiels:

- consentir aux pays surendettés qui sont engagés dans une restructuration effective les allègements de dette et les nouveaux financements nécessaires à leur développement économique;

- vu l'ampleur des déséquilibres des relations commerciales et financières de la Suisse avec le Tiers Monde, la Confédération devrait s'attaquer résolument aux causes (à celles qui se situent au sein des structures suisses) et, en attendant, les effets des mesures correctrices, recycler dans les pays débiteurs certains excédents de la balance suisse des opérations courantes.

De telles interventions permettraient à la Confédération de déterminer ellemême, dans l'intérêt bien compris de la population suisse, les modalités des ajustements indispensables. Si ceux-ci ne sont pas effectués (par la Suisse et par d'autres pays), la crise s'aggravera encore et des bouleversements d'une ampleur encore inconnue à ce jour pourraient se produire brusquement. Les effets de tels chocs seraient sans doute incontrôlables.

La réalisation de la politique esquissée ci-dessus serait facilitée par l'acceptation du principe d'une "conditionnalité réciproque» entre débiteurs et créanciers. Pour obtenir l'appui du Parlement et des citoyens, la Confédération devrait améliorer sa politique de l'information dans le domaine des relations économiques NordSud.

Notes:

1. Rapport du 12.3.84. Feuille fédérale 1984, Vol. I, pp. 853-897.

2. Postulat Reiniger du 6.10 .82 sur les risques inhérents à l'endettement international, cosigné par 25 conseillers nationaux. 
3. SIEBER H., «Internationale Schuldenkrise: eine Zwischenbilanz". Referat gehalten vor der Vereinigung Schweizerischer Kreditfachleute, Zürich, 24.9.85, $31 \mathrm{~S}$.

SOMMARUGA C., «Baker-Initiative: Die Sicht der Bundesbehörden». Einführungsreferat am Panel «Baker-Initiative" der Studiengesellschaft Schweiz-Dritte Welt, Bern, 26.2.86, $12 \mathrm{~S}$.

4. Voir notamment les exposés de Pierre Languetin à l'Assemblée générale de la Banque nationale suisse, 25.4 .86 et 24.4 .87 .

5. Réponse du 21.11.86 à la pétition «Pour que la faim ne soit le prix de l'endettement"/ "Gegen Schulden, die das Essen fressen", 8 p. La pétition était munie de 24.179 signatures.

6. Messages concernant la coopération technique et l'aide financière, le financement de mesures de politique économique et commerciale au titre de la coopération, ainsi que les Rapports semestriels sur la politique économique extérieure.

7. Réponse écrite, approuvée par le Conseil fédéral le 16.9.87, à l'interpellation Widmer du 20.3.87 concernant l'endettement international.

8. Postulat Longet du 11.6.87 «Aide au développement: conditions-cadre» cosigné par 27 Conseillers nationaux.

9. Appel déposé le 16.10.86. Après l'avoir examiné, la commission des pétitions du Conseil national a proposé un postulat qui invite le Conseil fédéral à présenter un rapport global concernant la politique commerciale de la Confédération envers le Tiers Monde.

10. Rapport sur les risques de l'endettement international, 12.3.84, p. 2.

11. Idem, p. 36.

12. Voir Annuaire Suisse-Tiers Monde 1986, pp. 62-67.

13. RAMONETI., «Fin du protectionnisme et maintien de l'austérité: Le Mexique sur les rails du néolibéralisme", Le Monde diplomatique, avril 1986, pp. 24-25.

14. Voir «La situation économique des pays en développement", Annuaire Suisse-Tiers Monde 1987, Revue, Chap. I, point 9.1.

15. SOLANO D., «Nouvelle stratégie des banques face à la dette du Tiers Monde", Industrie et développement international, septembre 1987, pp. 401-402.

16. Idem. D'autre part, la lettre-bilan publiée en septembre 1987 par l'Institute of International Finance (IFF), organisme qui rassemble officiellement les banques créancières du Tiers Monde, souligne encore davantage les réticences des banques. Voir aLe Tiers Monde accusé de ne pas jouer le jeu", Journal de Genève, 23.9.87.

17. Réponse à l'interpellation Widmer (cf. référence $N^{\circ} 7$ ), p. 1.

18. Lettre du 21.11.86, p. 5. (cf. référence $n^{\circ} 5$ )

19. Idem, p. 6.

20. Position rappelée dans la lettre de l'Ambassadeur de Suisse aux Etats-Unis au Directeur général du FMI, du 10.4.84. Feuille fédérale 1984, Vol. II, p. 1187.

21. Lettre du 21.11.86, pp. 2-4.

22. VIle session plénière de la CNUCED, Déclaration de la Délégation suisse au Comité IV (PMA), juillet 1987, p. 3.

23. Réponse à l'interpellation Widmer (cf. référence $N^{\circ} 7$ ), pp. 2-3.

24. World Debt Tables, External Debt of Developing Countries, 1986-87 Edition. Washington, The World Bank, 1987, p. xvi. Ce passage librement traduit se trouve dans le chapitre introductif almplementing the Consensus", pp. ix-xvii.

25. Idem, p. xiii.

26. Pour une présentation de ces politiques et une analyse de leurs imbrications, voir les articles de J. Forster et d'A. Brawand dans I'Annuaire Suisse-Tiers Monde 1982, pp. 181266. 
27. Message du Conseil fédéral concernant le financement de mesures de politique économique et commerciale au titre de la coopération internationale au développement, du 9.8.78, pp. 19-20.

28. Arrêtés fédéraux des $17.3 .66,18.3 .70$ et 20.6 .80 concernant la conclusion d'accords relatifs à des consolidations de dettes.

29. Cette politique aura généralement déjà été discutée lors de réunions du Groupe consultatif organisées par la Banque mondiale ou de la Table ronde parrainée par le PNUD.

30. Cf. article 10 a. de la loi sur la GRE et article 2, alinéa 3, de l'ordonnance. Dans leur demande de garantie, les requérants déclarent avoir pris connaissance des bases légales de la GRE.

31. La Confédération reprenait autrefois en son nom la part des créances consolidées non couvertes par la GRE. Le 14.1.81, le Conseil fédéral a décidé que les exportateurs devraient dorénavant supporter eux-mêmes le solde du risque.

32. La GRE avait versé pour 1309 millions de francs d'indemnités et récupéré 195 millions.

33. Rapport sur 'exercice annuel 1986 de la GRE, p. 7 et annexe C. Rappelons, en ce qui concerne le financement à l'exportation, que les banques réclament généralement aux exportateurs le remboursement de la part du montant consolidé non couverte par la GRE. Elles réclament aussi de plus en plus souvent la rétrocession de la part des intérêts impayés.

34. Voir précisions dans «Rééchelonnements de dettes au Club de Paris", Annuaire Suisse-Tiers Monde 1987, Revue, chap. I, point 9.8.

35. Réponse à l'interpellation Widmer (cf. référence $N^{\circ} 7$ ), p. 2.

36. Idem, question $\mathrm{N}^{\circ} 2$.

37. DIETHELM R., "Exportrisikogarantie braucht starke Medizin", Tages-Anzeiger, 10.6.87.

38. Idem. Voir aussi “Schleudergefahren für die ERG. Ein Ordnungspolitisches Dilemma», Neue Zürcher Zeitung, 29.7.87.

39. Réponse à l'interpellation Widmer (cf. référence $n^{\circ} 7$ ), p. 3.

40. «IWFMeltbank: Gläubiger Schweiz mitbetroffen", Tages-Anzeiger, 30.9.87.

41. Voir la 1e partie du texte: "Analyses et objectifs de la Confédération suisse".

42. Arrêté fédéral concernant la conclusion d'accords relatifs à des consolidations de dettes, du 20.6.80, art. 1er, al. 3.

43. D'autre part, les textes des accords bilatéraux de consolidation de dettes ne paraissent plus dans le Recueil des lois fédérales $(R O)$ depuis 1983. Ils peuvent être obtenus sur demande motivée, mais ils ne sont accompagnés d'aucun texte explicatif.

44. Cette section traite des contributions bilatérales et multilatérales gérées par l'OFAEE. Elle mériterait d'être complétée notamment par l'étude de cofinancements de la DDA destinés à soutenir des programmes d'ajustement structurel. Pour une réflexion générale sur ce thème, voir J. FRIEDEN, « La participation des agences de coopération bilatérales aux programmes d'ajustement des pays africains", Actes du Colloque sur les politiques d'ajustement structurel, Neuchâtel, 12-13 juin 1987.

45. Message concernant la continuation du financement de mesures de politique économique et commerciale au titre de la coopération internationale au développement, du 14.12.81, pp. 33-34.

46. DDA et OFAEE, Coopération au développement de la Confédération suisse. Rapport annuel 1986, p. 25.

47. Message concernant la continuation du financement de mesures de politique économique et commerciale au titre de la coopération internationale au développement, du 
19.2.86, pp. 44-48 et Rapport sur la politique économique extérieure 1986/2, du 14.1.87, chiffre 722.

48. Outre le financement des 15 accords mentionnés dans le texte, cette somme a servi à verser en 1981 un montant de 5,2 millions de francs sur un compte de bonification des intérêts ouvert par le FMl dans le cadre d'une de ses facilités additionnelles. L'utilisation du compte a permis de réduire le coût de prêts accordés aux pays à faible revenu.

49. Commission pour les questions conjoncturelles, "La balance suisse des paiements en 1986". Supplément de La Vie économique et du Bulletin mensuel de la BNS, septembre 1987.

50. L'excédent resterait considérable même si l'on pouvait corriger la statistique du commerce extérieur en tenant compte de l'origine réelle de certains produits (par ex. le pétrole brut) qui sont enregistrés par les douanes comme des marchandises en provenance des pays voisins. D'autre part, la statistique se limite au commerce des biens. Celui des services est encore plus déséquilibré.

51. Article 1er, alinéa 2 de la loi fédérale sur la garantie contre les risques à l'exportation, introduit dans la loi le 10.10.80.

52. Réponse à l'interpellation Widmer (cf. référence $N^{\circ} 7$ ): $4 e$ question et réponse aux pp. 4-5.

53. Cf. références $\mathrm{N}^{\circ} 8$ et 9 .

54. Conseil national, session d'été 1987. «Appel pour un commerce équitable avec le Tiers Monde» ( $N^{\circ} 86.68$ ). Rapport de la commission des pétitions du 27.3.87.

55. L'engagement de $\mathbf{4 0}$ millions de francs au cours des 4 prochaines années en faveur des pays les plus pauvres qui ont enregistré les déficits les plus importants dans leurs exportations de produits de base vers la Suisse, représente un bon geste, mais il n'est pas du tout à la hauteur de nos responsabilités!

56. Voir référence $\mathrm{N}^{\circ} 11$. 